\title{
Diferentes substratos na produção de mudas de mirtileiro
}

\author{
Different substrates in the production of blueberry seedlings
}

\section{Nara Cristina Ristow ${ }^{I}$ Luis Eduardo Correa Antunes ${ }^{\text {II }}$ Silvia Carpenedo ${ }^{\text {III }}$ Marcia Wulff Schuch ${ }^{\text {III }}$}

\section{RESUMO}

O objetivo desse trabalho foi avaliar o efeito da composição de diferentes substratos na produção de mudas de mirtileiro. Foram utilizadas mudas da cultivar 'Georgiagem', oriundas de multiplicação in vitro. Foram utilizados sete diferentes substratos, os quais são: T1 - Plantmax ${ }^{\circledR}(100 \%)$; T2 - Plantmax ${ }^{\circledast}+$ perlita $(1: 1) ;$ T3 - Solo + matéria orgânica + perlita (1:1:1); T4 - solo + casca de arroz (1:1); T5 - solo + composto industrial + vermiculita (1:1:1); T6 - casca de acácia + solo (1:1); T7 - acícula de pinus + solo - (1:2). O substrato acícula de pinus + solo, seguido pelos substratos Plantmax $^{\circledast}$, casca de acácia + solo e casca de arroz + solo promoveram maior acúmulo de massa seca da parte aérea e raízes. Os substratos solo + composto industrial + Perlita e Solo + composto industrial + vermiculita, com $\mathrm{pH}$ alcalino, apresentaram menor produção de massa seca e menores concentrações de Fe, Mn, Zn, N e P. Os substratos acícula de pinus, casca de acácia + solo, casca de arroz + solo e Plantmax ${ }^{\circledast}$ com ou sem perlita, mostraram-se os mais adequados para a produção de mirtileiro, cultivar 'Georgiagem'.

Palavras-chave: highbush, nutrição, 'Georgiagem', in vitro, Vaccinium.

\section{ABSTRACT}

The research aimed to evaluate the effect of the composition of different substrate on the production of blueberry seedlings. The seedlings used were highbush, 'Georgiagem' from in vitro multiplication. Seven different substrate were used: T1 - Plantmax ${ }^{\circledR}(100 \%) ;$ T2 - Plantmax ${ }^{\circledR}+$ perlita $^{-}$ (1:1); T3 - soil + composed industrial + perlita $(1: 1: 1) ;$ T4 soil + rice husks (1:1); T5 - soil + composed industrial + vermiculite (1:1:1); T6 - acácia bark + soil (1:1); T7 - pine needle mulch + soil $(1: 2)$. The substrate pine needle mulch + soil, followed by the substrate Plantmax ${ }^{\circledR}$, acácia bark + soil and soil/rice husks + soil, which promoted greater dry mass of roots and shoots. The substrate Soil + composed industrial + perlita and soil + composed industrial + vermiculite, $p H$ alkaline, showed lower production of dry matter and lower concentrations of Fe, Mn, Zn, N and P. Pine needle mulch + soil, soil + rice husks, acácia bark + soil and the substrate Plantmax $^{\circledast}$ with or without perlita $g$ were the most suitable $r$ for the production of blueberry seedlings, cultivar 'Georgiagem'.

Key words: highbush, nutrition, 'Georgiagem', in vitro, Vaccinium.

\section{INTRODUÇÃO}

O cultivo do mirtileiro (Vaccinium spp.), nativo da América do Norte, está em franca expansão em países da América do Sul, como Chile, Argentina e Uruguai (BAÑADOS, 2006). Aárea plantada de mirtileiro na América do Norte, Estados Unidos e Canadá teve um incremento de 30\% no período de 1992 a 2003 (STRIK \& YARBOROUGH, 2005).

O mirtilo atualmente é explorado comercialmente nos seis continentes. $\mathrm{O}$ crescimento das áreas plantadas se deve ao aumento do consumo, em função da população buscar nos últimos anos produtos saudáveis e com um alto potencial antioxidante (GIONGO \& BERGAMINI, 2003), benéficos a saúde.

'Embrapa Semiárido, CP 23, 56302-970, Petrolina, PE, Brasil. E-mail: ncristow@hotmail.com. Autor para correspondência. "Embrapa Clima Temperado, Pelotas, RS, Brasil.

IIIUniversidade Federal de Pelotas (UFPEL), Capão do Leão, RS, Brasil. 
Uma das principais dificuldades para a implantação da cultura é o desenvolvimento das mudas quando levadas para o campo, sendo o substrato importante para fornecer os nutrientes necessários ao desenvolvimento inicial das mudas.

Conforme SHELTON \& MOORE (1981), o substrato é um fator de grande importância na propagação de mirtileiro, que apresenta um sistema radicular muito superficial, sendo as raízes muito finas, não dispondo de pêlos radiculares. É muito sensível à compactação e à má drenagem do solo, devendo ser cultivado em solos ácidos, com pH entre 4 e 5,5, arenosos, franco-arenoso ou argilosos não muito profundos e de baixa fertilidade (BALLINGER, 1966). $\mathrm{O}$ mirtileiro pode ser cultivado, sem problemas aparentes, em solo com $\mathrm{pH}$ próximo a 6,0, desde que seja rico em matéria orgânica (HANSON \& HANCOCK, 2003). Deve-se dar atenção especial ao pH dos substratos, uma vez que é uma espécie que se desenvolve melhor em solos ácidos.

A matéria orgânica é um componente fundamental dos substratos, cuja finalidade básica, de acordo com CORDELL \& FILER JR. (1984), é aumentar a capacidade de retenção de água e nutrientes para o desenvolvimento das mudas. Com relação ao pH, os substratos devem apresentar valores dentro de uma faixa considerada adequada para o cultivo de plantas, pois valores inadequados, além de influenciar a disponibilidade de nutrientes (CARNEIRO, 1995), estão relacionados a desequilíbrios fisiológicos (WILSON, 1983).

A análise foliar vem sendo muito utilizada na quantificação do estado nutricional das culturas. A avaliação do estado nutricional das culturas pela diagnose foliar analisa determinadas folhas em períodos definidos da vida da planta, pois as folhas, de maneira geral, são os órgãos que refletem melhor o estado nutricional, isto é, respondem mais a variações no suprimento do nutriente (MALAVOLTA et al., 1989).

Tais fatos configuram ao tema grande importância para a realização de estudo, neste sentido, o conhecimento dos efeitos da composição do substrato nos teores foliares das mudas de mirtileiro poderá auxiliar na escolha da fonte e proporções de matéria orgânica a ser utilizada para compor os substratos na produção das mudas. Deste modo, o trabalho teve como objetivo avaliar o efeito da composição de diferentes substratos sobre a produção de mudas de mirtileiro, cultivar 'Georgiagem', oriundas de multiplicação in vitro.

\section{MATERIAL E MÉTODOS}

O trabalho foi desenvolvido em estufa no campo experimental da Embrapa Clima Temperado, Pelotas, RS. Foram utilizadas mudas da cultivar
'Georgiagem' (grupo Highbush), oriundas de multiplicação in vitro, com altura média entre 12 e $15 \mathrm{~cm}$.

As mudas foram transplantadas para vasos com capacidade para 6 Litros de substrato, utilizando sete diferentes substratos para a formação das mudas. Foram avaliados os substratos Plantmax ${ }^{\circledR}$ HT, perlita, matéria orgânica proveniente de resíduos industriais da região de Montenegro/RS, (composta por casca esgotada de acácia negra, polpa e sucos de frutas, resíduo de abatedouros, indústria de óleo de soja, cervejaria), vermiculita, casca de acácia, casca de arroz em decomposição proveniente de região arrozeira, solo/ acícula de pinus proveniente de varredura, solo (Planossolo Hidromórfico eutrófico solódico) proveniente da Embrapa Clima Temperado, misturados em diferentes combinações e proporções $(\mathrm{v} / \mathrm{v})$, conforme descritos a seguir: T1 - Plantmax ${ }^{\circledR}(100 \%)-$ (Testemunha), T2 - Plantmax ${ }^{\oplus}+$ perlita (1:1), T3 - solo + composto industrial + perlita (1:1:1), T4 - casca de arroz + solo (1:1), T5 - solo + composto industrial + vermiculita (1:1:1), T6 - casca de acácia + solo (1:1), T7 - solo/acícula de pinus (1:2). Foram realizadas quatro aplicações mensais por vaso de fertilizante $(250 \mathrm{ml})$, composto por sulfato de amônio (12\%), uréia (35\%), sulfato de potássio (10\%), sulfato de magnésio (10\%) e ácido fosfórico (10\%), a pH 2,8.

Foram avaliados os seguintes parâmetros: acúmulo de matéria seca da parte aérea (MSPA) e raiz (MSSR), análise química dos substratos antes e após os 120 dias da condução do experimento e a concentração foliar de nutrientes das mudas. As amostras dos substratos foram coletadas antes da implantação e após a avaliação destrutiva das mudas. As amostras para análise foliar e as interpretações dos resultados foram realizadas segundo a metodologia descrita por FREIRE (2006), sendo usada como base, pois não se tem conhecimento das necessidades nutricionais do mirtileiro na fase de muda. Os vasos foram mantidos em estufa com cortinas plástica nas laterais onde, aplicou-se, por meio de duas irrigações diárias via gotejamento, $200 \mathrm{~mL}$ de água por dia.

$\mathrm{O}$ delineamento estatístico adotado no experimento foi inteiramente casualizados, com sete tratamentos e quatro repetições onde a unidade experimental foi composta por cinco plantas. Os dados foram submetidos à análise de variância, e as médias, comparadas pelo teste de Scott-Knott em nível de 5\% de probabilidade de erro.

\section{RESULTADOS E DISCUSSÃO}

Ocorreram diferenças significativas no crescimento das mudas de mirtileiro cv. 'Georgiagem' submetidas aos diversos substratos, para todas as características avaliadas. Conforme tabela 1, com 
Tabela 1 - Médias da massa seca do sistema radicular (MSSR), massa seca da parte aérea (MSPA) das mudas de mirtileiro, cv. 'Georgiagem' em diferentes composições de substrato. Embrapa Clima Temperado, Pelotas/RS, 2011.

\begin{tabular}{lll}
\hline Tratamentos & MSPA $(\mathrm{g})$ & MSSR $(\mathrm{g})$ \\
\hline Plantmax $^{\circledR}$ & $33,02 \mathrm{~b}$ & $21,32 \mathrm{~b}$ \\
Plantmax $^{\circledR}+$ perlita & $23,53 \mathrm{~d}$ & $14,69 \mathrm{c}$ \\
Solo + composto industrial + perlita & $4,98 \mathrm{e}$ & $7,74 \mathrm{~d}$ \\
Casca de arroz + solo & $27,11 \mathrm{c}$ & $16,05 \mathrm{c}$ \\
Solo+composto industrial+vermiculita & $5,28 \mathrm{e}$ & $5,05 \mathrm{e}$ \\
Casca de acácia + solo & $21,39 \mathrm{~d}$ & $13,69 \mathrm{c}$ \\
Acícula de pinus + solo & $57,95 \mathrm{a}$ & $45,02 \mathrm{a}$ \\
CV $(\%)$ & 9,70 & 9,40 \\
\hline
\end{tabular}

* Médias seguidas pela mesma letra, na coluna, não diferem entre si pelo teste de Scott-Knott $(\mathrm{P}=0,05)$.

relação à produção de massa seca, o substrato acícula de pinus + solo foi superior aos demais tratamentos, tanto para a produção de massa seca das raízes $(45,02 \mathrm{~g})$, como para massa seca da parte aérea $(57,95 \mathrm{~g})$, seguido dos substratos Plantmax ${ }^{\circledR}$ e casca de arroz + solo.

Quanto ao Potencial Hidrogeniônico $(\mathrm{pH})$ (Tabela 2), os substratos contendo mistura de solo com composto industrial, perlita e vermiculita em sua composição, apresentaram os maiores valores de $\mathrm{pH}$, diferindo dos demais. Os substratos Plantmax ${ }^{\circledR}$, acícula de pinus + solo, casca de arroz + solo e Plantmax ${ }^{\circledR}+$ perlita obtiveram os menores valores. Estes resultados são corroborados por VERDONCK et al. (1981), relata que o material acumulado sob uma floresta de pinus (litter) apresenta valores de $\mathrm{pH}$ entre 3,9 e 5,5; as cascas entre 6,0 e 6,8; a perlita entre 6,5 e 7,2; a vermiculita entre 5,5 e 9,0. A cultura do mirtileiro exige solos mais ácidos $\left(4,2\right.$ a 5,5), sendo que Plantmax ${ }^{\circledR}$ e as combinações de acícula de pinus + solo, casca de arroz + solo e Plantmax ${ }^{\circledR}+$ perlita seriam as ideais, visto que o pH em níveis superiores, as plantas não se desenvolvem e apresentam sérios problemas de deficiência de Fe (BOUNOUS, 1996). Os melhores resultados relacionados à produção de massa seca foram observados em substratos com $\mathrm{pH}$ ácido.

O substrato acícula de pinus + solo destacou-se com relação ao alumínio, com resultado superior aos demais materiais, devido este substrato apresentar $\mathrm{pH}$ baixo, o que possibilita a presença e disponibilização de compostos de alumínio e manganês.

O teor de fósforo permaneceu elevado, apesar das perdas por lixiviação e pela adsorção das partículas do solo e ainda, pela absorção do sistema radicular durante o crescimento das mudas. O substrato Plantmax ${ }^{\circledR}$ combinado ou não com perlita apresentou maiores valores de fósforo (tabela 2). Conforme ABREU JR. et al. (2002), o aumento na disponibilidade de fósforo deve-se à presença do nutriente no adubo orgânico e aos aumentos do valor de $\mathrm{pH}$ e do teor de matéria orgânica. $\mathrm{O}$ aumento do $\mathrm{pH}$ do solo até próximo a 7,0 propicia maior disponibilidade de fósforo, uma vez que, em condições ácidas, ocorre reação do $\mathrm{H}_{2} \mathrm{PO}_{4}{ }^{-}$com as formas iônicas de ferro e alumínio, formando compostos

Tabela 2 - Composição química dos substratos após 120 dias de condução do experimento das mudas de mirtileiro (Vaccinium spp), cv. 'Georgiagem'. Embrapa Clima Temperado, Pelotas/RS, 2011.

\begin{tabular}{|c|c|c|c|c|c|c|c|}
\hline \multirow{2}{*}{ Tratamentos } & \multirow{2}{*}{$\begin{array}{c}\mathrm{pH} \\
\text { água }\end{array}$} & \multirow{2}{*}{$\begin{array}{c}\text { M.O. } \\
\%(\mathrm{~m} / \mathrm{v})\end{array}$} & K & \multirow[t]{2}{*}{$P$} & $\mathrm{Al}$ & $\mathrm{Ca}$ & $\mathrm{Mg}$ \\
\hline & & & -------m & & \multirow{2}{*}{$0,12 \mathrm{~b}$} & $\mathrm{cmol}_{\mathrm{c}} \mathrm{dm}^{-3}$ & \\
\hline Plantmax $^{\circledR}$ & $5,53 \mathrm{~b}$ & $8,25 \mathrm{a}$ & $376,5 b$ & $231,75 \mathrm{a}$ & & $14,7 \mathrm{c}$ & $8,05 \mathrm{a}$ \\
\hline Plantmax $^{\circledR}+$ perlita & $5,27 \mathrm{~b}$ & $8,37 \mathrm{a}$ & $299,5 \mathrm{c}$ & $174,75 b$ & $0,10 \mathrm{~b}$ & $18,97^{\mathrm{a}}$ & $3,35 \mathrm{~b}$ \\
\hline Solo + composto industrial + perlita & $7,37 \mathrm{a}$ & $5,14 \mathrm{c}$ & $158,5 \mathrm{e}$ & $131,25 \mathrm{c}$ & $0,00 \mathrm{~b}$ & $16,95 b$ & $2,22 \mathrm{c}$ \\
\hline Casca de arroz + solo & $4,87 \mathrm{c}$ & $6,12 \mathrm{~b}$ & $175,5 \mathrm{~d}$ & $105,50 \mathrm{~d}$ & $0,10 \mathrm{~b}$ & $4,47 \mathrm{e}$ & $2,32 \mathrm{c}$ \\
\hline Solo+comp. industrial +vermiculita & $7,42 \mathrm{a}$ & $3,97 \mathrm{~d}$ & $393,0 \mathrm{a}$ & $129,00 \mathrm{c}$ & $0,00 \mathrm{~b}$ & $15,0 \mathrm{c}$ & $3,30 \mathrm{~b}$ \\
\hline Casca de acácia + solo & $5,57 \mathrm{~b}$ & $5,13 \mathrm{c}$ & $78,5 \mathrm{f}$ & $14,75 \mathrm{f}$ & $0,02 \mathrm{~b}$ & $8,87 \mathrm{~d}$ & $1,30 \mathrm{~d}$ \\
\hline Acícula de pinus + solo & $4,57 \mathrm{c}$ & $5,42 \mathrm{c}$ & $82,0 \mathrm{df}$ & $51,50 \mathrm{e}$ & $0,45 \mathrm{a}$ & $3,87 \mathrm{e}$ & $1,57 \mathrm{~d}$ \\
\hline $\mathrm{CV}(\%)$ & 5,44 & 7,34 & 1,78 & 2,01 & 20,82 & 4,97 & 10,12 \\
\hline
\end{tabular}

* Médias seguidas pela mesma letra, na coluna, não diferem entre si pelo teste de Scott-Knott $(\mathrm{P}=0,05)$. 
de baixa solubilidade, além de maior adsorção do ânion por óxidos de ferro e alumínio presentes na fase sólida. A matéria orgânica, por sua vez, bloqueia os sítios de adsorção em óxidos de ferro e de alumínio do solo, diminuindo a capacidade de adsorção do $\mathrm{H}_{2} \mathrm{PO}_{4}$ (NOVAIS \& SMYTH, 1999).

Os maiores valores de magnésio foram observados nos substratos com presença de Plantmax ${ }^{\circledR}$ e vermiculita, em cuja composição esta incluída este macronutriente. O substrato acícula de pinus + solo, por sua vez, é um material pobre de nutrientes, apresentando somente teor de alumínio mais elevado. Substratos extremamente ácidos, com valores de $\mathrm{pH}$ inferiores a 4,5, são pouco férteis, por não reterem cátions como $\mathrm{K}^{+}, \mathrm{Ca}^{++}, \mathrm{e} \mathrm{NH}_{4}^{+}$(VAN DEN DRIESSCHE \& LECOURT, 1984; CARNEIRO, 1995). O máximo crescimento do mirtileiro, tanto cultivado em areia, como em solução nutritiva, é obtido com o uso de cerca da metade da concentração de nutrientes usados para as demais frutíferas. A extração anual de macronutrientes por uma planta adulta de mirtileiro ocorre na seguinte ordem: nitrogênio > cálcio > potássio > fósforo > magnésio(FREIRE, 2006).

Nas tabelas 3 e 4 são apresentados os efeitos dos substratos nas concentrações de micro e macronutrientes nas folhas das mudas. Os maiores teores de potássio nos substratos não influenciaram na concentração desse nutriente nas folhas, encontrando-se em excesso nas mudas. $\mathrm{O}$ magnésio apresentou comportamento semelhante ao cálcio em níveis dentro do normal para o desenvolvimento das mudas.

Os teores de nitrogênio e fósforo nos tratamentos solo + composto industrial + perlita e solo + composto industrial + vermiculita foram significativamente menores comparado aos demais substratos, os quais apresentavam $\mathrm{pH}$ acima de 7,0 e em níveis insuficiente para o desenvolvimento das mudas. Entretanto, os maiores teores de fósforo nos substratos não influenciaram na concentração desse nutriente nas folhas. O fósforo é muito importante nos solos ácidos. Isto se deve ao fato de que, apesar dos solos conterem grandes quantidades de fósforo total, a sua disponibilidade para as plantas é muito pequena, devido à tendência do fósforo em formar compostos de muito baixa solubilidade no solo (BISSANI et al., 2004). A adubação elevada com fósforo e o nível elevado do $\mathrm{pH}$ do solo podem induzir deficiências de zinco (MARSCHNER, 1995).

Segundo HANSON \& HANCOCK (1996), as deficiências de micronutrientes, não são comuns em mirtileiro, a menos que o pH do solo seja demasiadamente elevado. Os sintomas da deficiência do ferro são, geralmente, o primeiro indicador de solo $\mathrm{pH}$ impróprio, embora o $\mathrm{pH}$ influencie também na disponibilidade de $\mathrm{B}, \mathrm{Cu}, \mathrm{Mn}, \mathrm{Mo} \mathrm{e} \mathrm{Zn}$ às plantas. Para esta razão, a maioria de problemas com micronutrientes, podem ser adequados simplesmente ajustando o solo pH à escala apropriada.

O nutriente cobre apresentou pequena diferença na concentração foliar, mostrando-se em concentrações insuficiente para todos os tratamentos. De maneira geral, a elevação do $\mathrm{pH}$ do solo, o elevado teor de matéria orgânica e o excesso de N, P e Zn, favorecem o aparecimento da deficiência de cobre (MARSCHNER, 1995).

Observa-se uma diferença significativa no teor de manganês, onde os menores valores foram encontrados nos substratos de pH ácido (Tabela 4). Níveis altos de manganês nas plantas estão associados a solos de acidez elevada, como os detectados em alguns substratos do presente trabalho.

Tabela 3 - Concentração de macronutrientes nas folhas das mudas de mirtileiro (Vaccinium spp), cv. 'Georgiagem', em diferentes composições de substrato. Embrapa Clima Temperado, Pelotas/RS, 2011.

\begin{tabular}{|c|c|c|c|c|c|}
\hline & $\mathrm{N}$ & $\mathrm{P}$ & $\mathrm{K} * *$ & $\mathrm{Ca}$ & $\mathrm{Mg}$ \\
\hline Plantmax $^{\circledR}$ & $15,2 \mathrm{a}$ & $0,9 \mathrm{a}$ & 10,5 & $5,0 \mathrm{c}$ & $1,7 \mathrm{~b}$ \\
\hline Plantmax $^{\circledR}+$ Perlita & $15,1 \mathrm{a}$ & $1,0 \mathrm{a}$ & 12,3 & $6,6 \mathrm{a}$ & $1,9 \mathrm{a}$ \\
\hline Solo + Composto industrial + Perlita & $11,3 \mathrm{~b}$ & $0,6 \mathrm{~b}$ & 11,4 & $5,7 \mathrm{~b}$ & $1,5 \mathrm{~b}$ \\
\hline Casca de arroz + Solo & $16,4 \mathrm{a}$ & $1,0 \mathrm{a}$ & 10,2 & $4,9 \mathrm{c}$ & $1,6 \mathrm{~b}$ \\
\hline Solo+Composto industrial+Vermiculita & $11,6 \mathrm{~b}$ & $0,6 \mathrm{~b}$ & 12,3 & $4,6 \mathrm{c}$ & $1,7 \mathrm{~b}$ \\
\hline Casca de acácia + Solo & $15,7 \mathrm{a}$ & $0,9 \mathrm{a}$ & 11,0 & $5,9 \mathrm{~b}$ & $1,7 \mathrm{~b}$ \\
\hline Acícula de pinus + Solo & $15,0 \mathrm{a}$ & $0,9 \mathrm{a}$ & 1,0 & $6,7 \mathrm{a}$ & $1,7 \mathrm{~b}$ \\
\hline $\mathrm{CV}(\%)$ & 9,02 & 11,48 & 27,43 & 7,07 & 6,97 \\
\hline Faixa de interpretação - Normal & $18,0-21,0$ & $1,2-4,0$ & $3,5-6,5$ & $4,0-8,0$ & $1,2-2,5$ \\
\hline
\end{tabular}

* Médias seguidas pela mesma letra, na coluna, não diferem entre si pelo teste de Scott-Knott $(\mathrm{P}=0,05)$. 
Tabela 4 - Concentração de micronutrientes nas folhas das mudas de mirtileiro (Vaccinium spp), cv. 'Georgiagem', em diferentes composições de substrato. Embrapa Clima Temperado, Pelotas/RS, 2011.

\begin{tabular}{lccccc}
\hline & Fe & Mn & Zn & Cu & B \\
Tratamentos & & & & \\
& & & & \\
& $121,50 \mathrm{~b}$ & $446,25 \mathrm{a}$ & $9,00 \mathrm{c}$ & $2,50 \mathrm{a}$ & $149,00 \mathrm{a}$ \\
Plantmax $^{\circledR}$ & $177,00 \mathrm{a}$ & $182,25 \mathrm{~b}$ & $13,00 \mathrm{~b}$ & $2,25 \mathrm{a}$ & $122,50 \mathrm{~b}$ \\
Plantmax $^{\circledR}+$ Perlita & $64,00 \mathrm{~d}$ & $65,00 \mathrm{~b}$ & $6,75 \mathrm{c}$ & $1,25 \mathrm{~b}$ & $125,75 \mathrm{~b}$ \\
Solo + Composto industrial + Perlita & $98,50 \mathrm{c}$ & $485,00 \mathrm{a}$ & $17,25 \mathrm{a}$ & $1,25 \mathrm{~b}$ & $116,75 \mathrm{~b}$ \\
Casca de arroz + Solo & $76,00 \mathrm{~d}$ & $39,00 \mathrm{~b}$ & $8,25 \mathrm{c}$ & $1,75 \mathrm{~b}$ & $121,00 \mathrm{~b}$ \\
Solo+Composto industrial+Vermiculita & $109,50 \mathrm{c}$ & $327,50 \mathrm{a}$ & $9,50 \mathrm{c}$ & $1,25 \mathrm{~b}$ & $118,00 \mathrm{~b}$ \\
Casca de acácia + Solo & $148,25 \mathrm{~b}$ & $501,25 \mathrm{a}$ & $9,50 \mathrm{c}$ & $3,50 \mathrm{a}$ & $68,25 \mathrm{c}$ \\
Acícula de pinus + Solo & 19,70 & 33,44 & 15,92 & 42,34 & 10,28 \\
CV (\%) & $81-199$ & $51-349$ & $15-30$ & $11-20$ & $31-69$ \\
Faixa de interpretação - Normal & & & & \\
\hline
\end{tabular}

* Médias seguidas pela mesma letra, na coluna, não diferem entre si pelo teste de Scott-Knott $(\mathrm{P}=0,05)$.

Com relação aos substratos, a concentração de boro apresentou valores acima do normal com exceção ao substrato acícula de pinus + solo, que se encontrava em teores normais. Já para as concentrações de zinco nas folhas o substrato solo + composto industrial + perlita, apresentou concentrações insuficientes, o substrato Casca de arroz + Solo em concentrações normais e os demais substratos em concentrações abaixo do normal.

Com relação ao ferro os substratos solo + composto industrial + perlita e solo + composto industrial + vermiculita apresentaram valores abaixo do normal para o desenvolvimento das mudas. Segundo MARSCHNER (1995), a elevação do pH, o excesso de matéria orgânica, as elevadas concentrações de $\mathrm{P}, \mathrm{Cu}, \mathrm{Mn}$ e $\mathrm{Zn}$ e o encharcamento do solo são fatores que induzem à carência de ferro.

Com exceção dos substratos solo + composto industrial + perlita e solo + composto industrial + vermiculita, durante o período de avaliação, nenhum sintoma visual de deficiência ou de toxidez causada pela falta ou excesso de nutrientes nos substratos foi observado. Os mesmos substratos apresentaram os menores valores de MSPAe MSSR, e menores concentrações de Fe, Mn, Zn, N e P.

\section{CONCLUSÃO}

O substrato acícula de pinus + solo, seguido pelos substratos Plantmax ${ }^{\circledR}$, casca de acácia + solo e casca de arroz + solo promovem maior crescimento da parte aérea e raízes em mudas de mirtileiro.

Os substratos solo + composto industrial + perlita e solo + composto industrial + vermiculita apresentam $\mathrm{pH}$ alcalino, menor crescimento da parte aérea e das raízes e, menores concentrações de Fe, $\mathrm{Mn}, \mathrm{Zn}, \mathrm{N}$ e $\mathrm{P}$ em mudas de mirtileiro.
Os substratos acícula de pinus, casca de acácia + solo, casca de acácia + solo e Plantmax ${ }^{\circledR}$ com ou sem perlita são substratos adequados para a produção de mirtileiro, cultivar 'Georgiagem'.

\section{AGRADECIMENTOS}

Os autores agradecem à Fundação de Amparo à Pesquisa do Estado do Rio Grande do Sul (FAPERGS), Conselho Nacional de Desenvolvimento Científico e Tecnológico (CNPq) e Coordenação de Aperfeiçoamento de Pessoal de Nível Superior (CAPES) pela concessão do auxílio financeiro e de bolsas de pesquisa.

\section{REFERÊNCIAS}

ABREU JR. et al. Carbono, nitrogênio, fósforo e enxofre em solos tratados com composto de lixo urbano. Revista Brasileira de Ciência do Solo, v.26, p.769-780, 2002. Disponível em: <http://sbcs.solos.ufv.br/solos/revistas/v26n3a22.pdf>. Acesso em: 20 jan. 2009.

BANÃDOS, M.P. Blueberry production in South América. Acta Horticulturae (ISHS), n.715, p.165-172, 2006. Disponível em: <http://www.actahort.org/books/715/715_24.htm>. Acesso em: 27 fev. 2009 .

BALLINGER, W.E. Soil management, nutrition and fertilizer practices. In: ECK, P.; CHILDRES, N. [Ed.]. Blueberry culture. Brunswick: Rutgers University, 1966. p.132-178.

BISSANI, C.A. et al. Fertilidade dos solos e manejo da adubação de culturas. Porto Alegre. Gênesis, 2004. 328p.

BOUNOUS, G. Piccoli frutti. Bologna: Edagricole, 1996. $434 \mathrm{p}$.

CARnEIRO, J.G.A. Produção e controle de qualidade de mudas florestais. Curitiba: Campos UENF, UFPR/FUPEF, 1995. $451 \mathrm{p}$.

CORDELL, C.E.; FILER Jr., T.H. Integrated nursery pest management. In: LANDZ, C.W. (Ed.). Southern pine 
nursery handbook. Atlanta: USDA. Forest Service, Southern Region, 1984. p.1-17.

FREIRE. C.J. da S. Nutrição e adubação para mirtilo. In: RASEIRA, M. do C.; ANTUNES, L.E.C. (Ed.). A cultura do mirtilo. Pelotas: Embrapa Clima Temperado, 2006. p.60-74. (Embrapa Clima Temperado, Sistemas de produção n.8).

GIONGO L.; BERGAMINI A. Breeding objectives for raspberry and highbush blueberry worldwide. Frutticoltura, v.65, n.11, p.39-44, 2003

HANSON, E.; HANCOCK, J. Managing the nutrition of highbush blueberries. In: JORNADA DE ARÁNDANOS - PRODUCCION EN ARGEntinA, 2003, Buenos Aires. Anales... Buenos Aires: FAUBA, 2003. CD-ROM.

HIGASHI, E.N. et al. Propagação vegetativa de Eucalyptus: princípios básicos e sua evolução no Brasil. Circular técnica IPEF, n.192, p.14, 2000.

MARSCHNER, H. Mineral nutrition of higher plants. 2.ed. San Diego, CA: Academic, 1995. 902p.

MALAVOLTA, E. et al. Avaliação do estado nutricional das plantas: princípios e aplicações. Piracicaba: Associação
Brasileira para Pesquisa da Potassa e do Fosfato, 1989. 201p.

NOVAIS, R.F.; SMYTH, T.J. Fósforo em solo e planta em condições tropicais. Viçosa: Universidade Federal de Viçosa, 1999. 399p.

SHELTON, L.L.; MOORE, J.N. Highbush blueberry propagation under southern U.S. climatic conditions. HortScience, v.16, n.3, p.320-321, 1981 .

STRIK, B.C.; YARBOROUGH, D. Blueberry production trends in North America, 1992 to 2003, and predictions for growth. HortTecnology, v.15, n.2, p.391-398, 2005.

VAN DEN HEEDE, A.; LECOURT, M. El estaquillado: guia práctica de multiplicación de las plantas. Madrid Mundi-Prensa, 1989. $171 \mathrm{p}$.

VERDONCK, O. de et al. The influence of the substrate to plant growth. Acta Horticulturae, Substrates in Horticulturae, v.126, p.251-258, 1981.

WILSON, C.G S. Tomato production in bark substrates. Acta Horticulturae, v.150, p.271-276, 1983. Disponível em: <http://www.actahort.org/books/150/150_29.htm>. Acesso em: 20 dez. 2008 